\title{
A Neurofunctional Evaluation Strategy for Presurgical Selection of Temporal Lobe Epilepsy Patients
}

\author{
Lilia María Morales Chacón, MD, PhD, Carlos Sánchez Catasús, PhD, Juan E. Bender, MD, Jorge Bosch Bayard, PhD, \\ María E García, MS, Ivan García Maeso, MD, Lourdes Lorigados Pedre, PhD, Bárbara Estupiñán Diaz, MD, \\ Otto Trápaga Quincoses, MD, Margarita Báez Martín, MD, Abel Sánchez Coroneaux, LPN, Digna Pérez Madrigal, BSN, \\ Miriam Guevara, BSN, Marilyn Zaldívar Bermúdez, Ángel Águila
}

\begin{abstract}
Introduction Temporal lobe epilepsy (TLE) is the prototype of a surgically correctable syndrome. Successful surgical outcomes depend on a thorough presurgical evaluation aimed primarily at identifying the epileptogenic zone.

Objective Describe the noninvasive presurgical selection and evaluation strategy for TLE patients introduced at the International Neurological Restoration Center (CIREN) in Havana, Cuba, and evaluated between 2001 and 2006 for its accuracy in identifying candidates for non-lesional resection surgery.

Methods Ictal onset electrographic patterns of 1,679 seizures in 72 patients with drug-resistant partial epilepsy, obtained through longterm scalp Video EEG (V-EEG) monitoring, were evaluated. The correlation between the V-EEG-defined epileptogenic zone and the dysfunction shown by single photon emission computed tomography (ictal and interictal brain SPECT) and nuclear magnetic resonance spectroscopy (MRS) was established.
\end{abstract}

Results V-EEG monitoring determined that $44.4 \%$ of evaluated patients had complex partial temporal lobe seizures. Identification

\section{INTRODUCTION}

About one-third of patients with symptomatic or cryptogenic focal epilepsy do not respond well to antiepileptic drugs (AEDs). For many of these patients, surgery is an effective and potentially safe therapeutic alternative, yet surgical management of refractory epilepsy is still underused. The first controlled study of temporal lobe epilepsy (TLE) surgery, reported in 2001, showed statistically significant advantages of surgery over drug therapy in terms of both seizure evolution and patients' quality of life.[1] A more recent study demonstrated that $44.6 \%$ of postoperative patients were seizure-free after 4.8 years, compared to only $4.3 \%$ of patients receiving drug therapy.[2]

Nevertheless, there is a reported 15-to-20-year lag in patient referrals to surgery programs.[3-8] The use of TLE surgery is mainly constrained by the possibility of surgical failure due to incomplete or erroneous resection of the epileptogenic zone (EZ). [9-11] Thus, improved presurgical evaluations aimed at correct EZ identification are needed.

The challenge is finding a method for precisely defining epileptogenicity. Several diagnostic tests with varying degrees of complexity and technical difficulty have been developed toward this end; however, there is no consensus about which of them can best define the EZ with a reasonable cost-benefit ratio.[12] These tests include magnetic resonance imaging (MRI), single photon of patients with medial temporal epilepsy (MTE) increased as a result of lateralization and localization of the dominant mean ictal pattern frequency $(5.56 \pm 1.31 \mathrm{~Hz})$ during the period of maximum spectral power VARETA localization of an ictal epileptiform activity source coincided with the epileptogenic zone in all TLE patients who subsequently underwent a successful temporal lobectomy. Semiquantitative analysis of ictal and interictal brain SPECT images, as well as metabolic ratios measured by MRS, combined with V-EEG findings, enabled localization/lateralization of the epileptogenic zone in TLE patients whose MRIs were normal or showed bilateral structural abnormalities.

Conclusions Confirmation of correct localization/lateralization of the epileptogenic zone following successful surgical outcomes in selected TLE patients led CIREN to develop a surgical treatment strategy for patients in Cuba with drug-resistant temporal lobe epilepsy. This strategy offers an appropriate, cost-effective treatment alternative for developing countries like Cuba, with the benefit of significantly improving TLE patients' quality of life.

Keywords: Epilepsy, temporal lobe, surgery, EEG, SPECT, MRI

emission computed tomography (SPECT), magnetic resonance spectroscopy (MRS), positron emission tomography (PET), and video electroencephalographic monitoring (V-EEG).

Advances in the development and application of these techniques during the last decade contributed to a better understanding of the brain's functional anatomy but did not substantially improve the accuracy of EZ localization. Even high-resolution MRI cannot always identify hippocampal sclerosis, the main neurological disease underlying TLE. Studies have shown that the EZ is unilateral with respect to the lesion in $65 \%$ of patients with unilateral hippocampal sclerosis, contralateral in $4 \%$ of cases, and is not localized or is bitemporal in $31 \%$ of patients.[13-24] For this latter group, the need for invasive monitoring during presurgical evaluation could be reduced if EZ localization were estimated in the clinic, taking into account the results of functional imaging tests.

This study evaluated V-EEG ictal onset localization recorded with scalp electrodes, using spectral analysis of the ictal EEG signal combined with source localization methods, as well as the correlation between these and functional neuroimaging techniques, for presurgical evaluation of TLE patients.

\section{METHODS}

Sample. Between 2001 and 2006, 72 patients with refractory complex partial epileptic seizures of presumed temporal origin 
were evaluated as candidates for surgical treatment. Their average age was $35.6 \pm 7.18$ years and duration of epilepsy was $22.75 \pm 9.22$ years. All subjects were admitted to the International Neurological Restoration Center's (Centro Internacional de Restauración Neurológica - CIREN) V-EEG Telemetry Unit in Havana, Cuba. The presurgical evaluation program protocol included a full clinical history, and full general physical and neurological examinations, as well as neuropsychological and neuropsychiatric evaluations, MRI (1.5 T Magnetom Symphony equipment), interictal and ictal brain SPECT, and $\mathrm{H}^{+}$MRS. Gradual AED tapering was applied and stopped when patients registered three partial or one tonic-clonic seizure per day.

Written informed consent was obtained from participating patients and their families. The research protocol was approved by the CIREN Ethics Committee.

\section{Evaluation of Video EEG combined with spectral analysis} and EEG source localization analysis. A continuous $V$-EEG monitoring system (STELLATE Video EEG Digital Recording System with Harmonie software, Canada) was used. Electrodes were attached using the 10-20 International System, including the following additional scalp electrodes: zygomatic (Cg1-2), true anterior temporal (T1-2) and supraorbital (SO1-2). Additionally, Electrocardiogram (ECG) and Electroocculogram (EOG) recordings were obtained. Patients admitted to the Video EEG Telemetry Unit were monitored around-the-clock until a sufficient number of seizures were recorded with adequate visualization and EEG quality.

Recorded seizures were classified in two categories based on the ictal onset EEG pattern: (a) temporal (localized in the temporal lobe) or (b) non-temporal. The criteria used to establish localization was the maximum amplitude observed in the referential montage, complemented by voltage maps of electrical brain activity. During each seizure, the following ictal activity patterns were defined: rhythmic activity (alpha, theta or delta frequencies); arhythmic activity; rapid paroxystic activity (within the beta frequency range); repetitive epileptiform activity (no less than three repetitive point discharges); suppression (reduction $>50 \%$ of the background amplitude or $<10 \mu \mathrm{V}$ ).

For statistical analysis, the electrodes involved at the electrographic ictal onset were grouped in the temporal zone (T1-2, Cg1-2, F7-F8, T3-4, T5-6), and the rest of the electrodes were placed in the non-temporal zone. A Chi-square test $\left(x^{2}\right)$ was used to analyze the dependency relationship between the electrodes involved at ictal onset and the categories defined. Significant differences between the defined categories were estimated using Student's t-test for independent samples and $p<0.05$.

In an effort to improve localization in patients with seizures categorized as temporal, spectral analysis of the electroencephalographic ictal pattern was performed using all recording channels, with emphasis on the temporal electrodes, using the Harmonie Software EEG Compressed Spectral Analysis (CSA) function.

The VARETA method was used in the time domain to analyze EEG sources in the ictal onset zone.[25] Recording windows were selected that guaranteed a minimum analysis period ranging from 12 seconds before to 36 seconds after the visually- defined electrographic ictal onset. The clinical ictal onset was not included.

2. Correlation between the V-EEG defined ictal onset zone and the dysfunction shown in the functional imaging (SPECT and MRS) studies, complemented by quantitative analysis. For this study, a subset of 18 patients who met the following criteria were selected from the original sample:

- Typical complex partial TLE seizures defined by ictal V-EEG recordings.

- Normal MRI images or images showing bilateral hippocampal sclerosis (presence of hippocampal atrophy and increased signal intensity), obtained from a report by two radiologists who had no knowledge of patients' clinical status.

- Surgical resection involving the temporal lobe (hippocampus, amygdala, parahippocampus and temporal neocortex resection) using standard electrocorticography-guided temporal resection procedure.

- Engel Class $1 \mathrm{~A}$ postsurgical evolution (completely seizurefree since surgery).

- Patients with 1-3 years postsurgical evolution.

The SMV DST XLi Sopha Medical Vision (France) double head SPECT system was used to obtain images. A semi-quantitative analysis of cerebral perfusion was done in the following regions of interest (ROI) in both brain hemispheres: lateral temporal region, mesial, basal lobes, and cerebellum. These studies were done in interictal and ictal states, the latter during V-EEG monitoring.

For MRS, spectra were obtained using a single voxel spectroscopy (SVS) acquisition protocol based on a spin-echo sequence (TR 1500 msec, TE $135 \mathrm{msec}, 512$ acquisitions). A $1 \times 1 \times 3$-centimeter $\left(3 \mathrm{~cm}^{3}\right)$ volume of interest (VOI), localized at the hippocampus level, was used. Absolute concentrations of N-acetyl aspartate (NAA), choline (Cho), and creatinine ( $\mathrm{Cr}$ ) metabolites in both hippocampuses were measured, as well as the following proportions: NAA/Cr and NAA/ Cho, Cho/Cr and NAA/Cr+Cho.

In order to test the hypothesis that the variables evaluated in the SPECT and MRS studies had lateralizing value, we created a difference variable that established the difference value between the right and left hemispheres, which, in turn, allowed for creation of a second right/left classification variable. A 95\% confidence interval was established. The hypothesis was then tested and considered confirmed if the test showed significant separation and no overlapping between the imaging variable values for each hemisiphere.

Concordance between lateralization/localization and subsequent resection was evaluated for each modality. Definition of the epileptogenic zone was deemed correct when the patient was seizurefree one year after resection. The correlation between correct EZ definition and lateralization identified by V-EEG, MRS and brain SPECT was established. Associations were made according to Fischer's exact test $(2 * 3,2$ tails; $p<0.05)$. Correct lateralization was defined as the surgically resected side in patients who were seizure free for a minimum postoperative period of one year.

\section{RESULTS}

1. Evaluation of Video EEG combined with spectral analysis and EEG source localization analysis. Continuous V-EEG 
monitoring resulted in evaluation of an average of 16.2 seizures/ patient in $11.3 \pm 4.12$ days. Based on the ictal onset $V$-EEG pattern, 421 seizures in 32 patients were categorized as temporal. This ictal onset pattern topography was not found in 1,009 seizures in 26 patients, which were classified as non-temporal. A statistically significant dependency correlation was found between the electrodes involved at ictal onset and the categories defined $\left(x^{2}(1)=17.54 ; p<0.01\right)$. In five patients, $V-E E G$ showed habitually reported seizures with no electrographic change associated with behavior, and these were classified as non-epileptic seizures.

This study confirmed that variability in the ictal pattern is independent of the topography, and also demonstrated that a dominant mean frequency can be distinguished in the theta range, which differentiates patients with temporal seizures from patients whose seizures have a different topography. This distinction was not affected by the presence of complex partial seizures, usually while awake, and the absence of special seizures.

Topographical analysis of the dominant ictal frequency classified $52.7 \%$ of seizures as temporal with a mesial focus (Figure 1, VEEG), and two predominant or dominant ictal frequencies in the ictal pattern were identified by CSA. Topographic localization of the dominant mean frequency $(5.56 \pm 1.31 \mathrm{~Hz})$, determined by spectral analysis of the EEG, predicted a mesial temporal epilepsy (MTE) diagnosis in $78.94 \%$ of patients, compared to $47.34 \%$ diagnosed by visual examination; however, behavioral signs alone provided no useful information for making the distinction between MTE and neocortical temporal epilepsy (NTE).

The VARETA distributed source localization method was used in the time domain to confirm the ictal onset zone defined by visual and spectral analysis in patients who underwent surgery. This enabled determination of an ictal epileptiform activity source that coincided in lateralization and localization with the EZ in all TLE patients who underwent successful temporal lobectomies, thereby establishing concordance between the ictal onset zone defined by scalp V-EEG, the activity source defined by VARETA, and the EZ (Figure 1, VARETA).

2. Correlation between the V-EEG defined ictal onset zone and the dysfunction shown in the functional imaging (SPECT and MRS) studies, complemented by quantitative analysis. Temporal lobe hypoperfusion in the interictal state was observed in $100 \%$ of the 18 evaluated patients--on the left side in $56.25 \%$, on the right side in $43.7 \%$, and extended to the frontal lobe in $50 \%$. A discriminating analysis using the ROI measurements evaluated found that perfusion changes in the lateral aspect of the temporal

Figure 1: Summary of Presurgical Evaluation Strategy for TLE Patients Combining V-EEG, CSA, VARETA, MRS and Brain SPECT

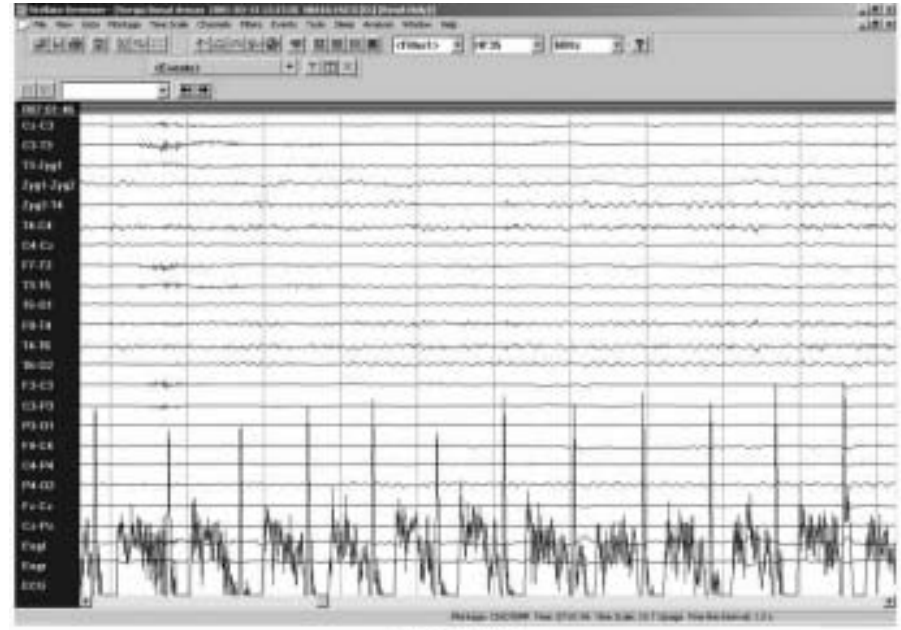

V-EEG

Right

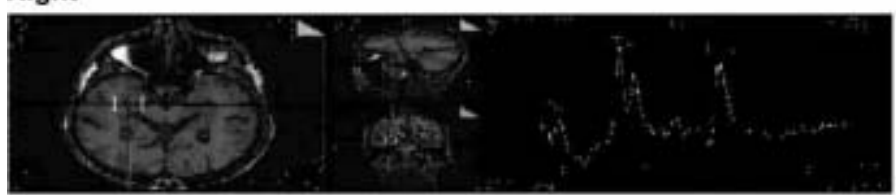

Left

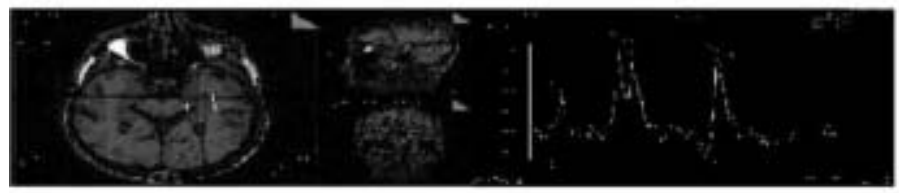

MRS
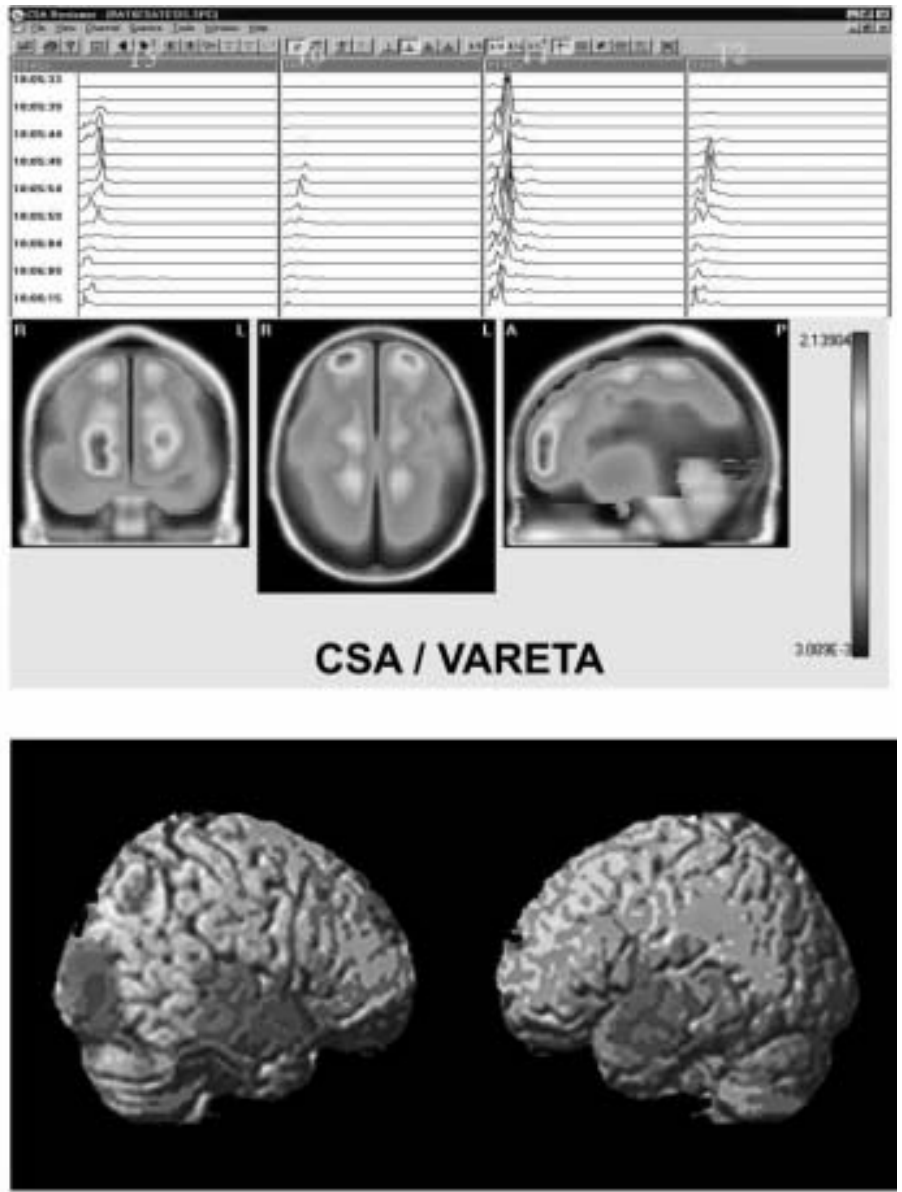

Brain SPECT 
region had the highest lateralization values. These were ipsilateral on the surgery side in $87.4 \%$ of patients $\left(x^{2}(2)=8 ; p<0.0001\right)$. Also, an inter-hemispheric perfusion asymmetry ratio of $13 \%$ and $28 \%$ was found in patients with left and right TLE, respectively. Hyperperfusion in the mediolateral aspect of the temporal lobe ipsilateral to the epileptogenic zone was found in $100 \%$ of cases examined with ictal SPECT (Figure 1, SPECT). Ictal studies have reported sensitivity ranging from $73.4 \%$ to $97 \%$, with a positive predictive value of $94 \%$.[26,27]

Discriminating analysis using V-EEG lateralization as a grouping variable found a statistically significant model, Wilks Lambda $0.224 \mathrm{~F}(3,8)=9.21 \mathrm{p}<0.0056$, which used $\mathrm{Cho} / \mathrm{Cr}(\mathrm{p}<0.003)$, $\mathrm{NAA} / \mathrm{Cr}(\mathrm{p}<0.001)$ and NAA/Cho+Cr $(p<0.03)$ ratios as influence variables for the metabolic data yielded by MRS, and found that lateralization was correct in $53.8 \%$ of patients.

Our analysis showed a $21.2 \%$ increase in the $\mathrm{Cho} / \mathrm{Cr}$ ratio and a $16.4 \%$ decrease in the NAA/Cr ratio in the hippocampus ipsilateral to the EZ compared to the contralateral hippocampus (Figure 1, MRS). Statistically significant associations were found between ictal V-EEG lateralization and SPECT $\left(x^{2}(1)=4.8 ; p=\right.$ $0.02)$; between ictal V-EEG and MRS $\left(x^{2}(2)=8 ; p=0.01\right)$, and between MRS and SPECT $\left(x^{2}(2)=5.8 ; p=0.05\right)$. This study found that ictal $V-E E G$ yielded the highest percentage of correct lateralization (100\%), followed by SPECT and the Cho/Cr and NAA/Cr metabolic ratios yielded by MRS (Figure 2).

\section{DISCUSSION}

Most studies evaluating the use of functional and structural imaging for lateralization of the EZ use EEG results as a reference. In our study, we also used surgical outcomes with a minimum one-year follow-up period of satisfactory evolution, and we evaluated patients whose MRI scans revealed no morphological lateralization. The results clearly demonstrated that detection of hippocampal lateralizing spectroscopic profiles is not necessarily accompanied by signs of lateralization (atrophy) in the morphological image.
Analysis of the relationship between information provided by V-EEG and the various functional imaging techniques for defining the epileptogenic zone in a group of patients who underwent temporal lobectomy showed that information yielded by the functional studies made successful surgery possible without the need for invasive tests, even in cases for which MRI scans were normal or not lateralizing. Most studies with similar designs reported in the literature use mainly MRI localization,[2831] whereas we based our findings on the gold standard of localization, that is, seizure-free after one year of postsurgical evolution.

In our evaluation of V-EEG combined with spectral analysis and EEG source localization analysis, three types of seizure pattern were seen in our sample: rhythmic pattern (theta, alpha and delta), rapid paroxistic activity, and repetitive epileptiform activity, which were not mutually exclusive. Ictal pattern analysis yielded the presence of inter-seizure variability in $40.8 \%$ of cases studied, expressed as a pattern composed of different ictal frequencies, even in seizures recorded in the same patient.

No dependency relationship in this variability was found in the two groups studied (temporal vs. non-temporal). It is important to take the ictal pattern variability into account when formulating an EZ hypothesis. To date, we know of no studies that have evaluated this phenomenon in patients with focal seizures of different localization.

In this study, using additional scalp electrodes for greater temporal lobe coverage, a statistically significant correlation was found between the rhythmic pattern at the theta frequency and seizures of temporal lobe origin. The ictal onset frequency of non-temporal seizures was statistically different from that of temporal seizures; rapid frequencies in the alpha range were verified during extratemporal seizures, particularly in frontal-lobe seizures, which may indicate intrinsic cortical circuit differences, as suggested by other authors.[32,33]

Figure 2: Correlation between Ictal V-EEG, SPECT and MRS Lateralization, and Surgical Resection Laterality



Ictal V-EEG vs. Resection. $X^{2}(1)=14 p=0.001$ Interictal

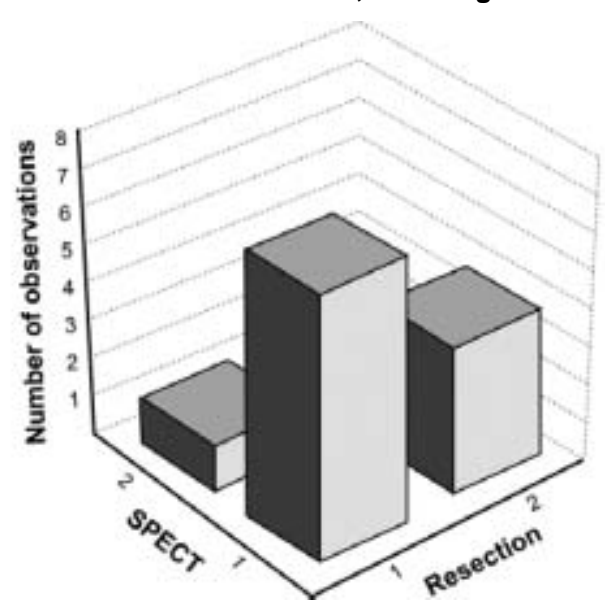

SPECT vs. Resection. $X^{2}(2)=13 p=0.015$

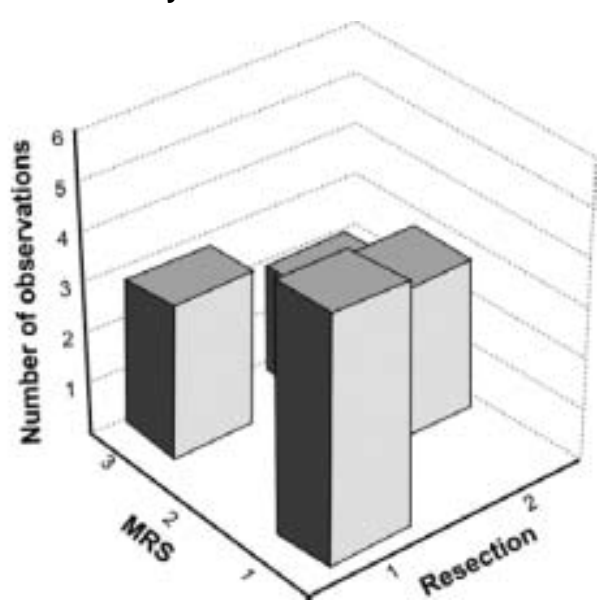

MRS vs. Resection. $X^{2}(2)=7.93 p=0.018$

$x$ axis: resection side, $y$ axis: technique used, 1: Left 2: Right 3: Bilateral 
Previous studies suggest that the frequency and spatial extension of the electrographic ictal onset are associated with the anatomical connections, while the underlying pathology determines its morphology, which predicts postsurgical evolution and indicates that the ictal electrical discharge is determined by a complex interaction between anatomical localization and the underlying pathology.[21,34 ]

Most ictal pattern studies reported in the literature do not include a quantitative analysis due to the technical difficulties involved making such an analysis.[35] In our study, compressed spectral analysis (CSA) of ictal activity was used, and ictal activity sources were estimated using distributed inverse solutions.

Different EEG spectral changes in patients with complex partial seizures have been described.[21] The most frequent pattern is characterized by a global attenuation of background activity, with no topographic value, followed by the appearance of rhythmic frequencies containing localizing information.[36]

In an analysis of these lateralizing rhythmic patterns, Risinger et al. found a high correlation between the ictal pattern at $5 \mathrm{~Hz}$ or faster frequencies in sphenoidal and/or temporal localization, and the presence of ipsilateral temporomesial ictal onset. This pattern is very specific but, unfortunately, it has a $40-50 \%$ sensitivity.[37] This finding was confirmed by a study of ictal pattern frequency differentiation in lateral vs. mesial temporal seizures, which found that the former had a typical frequency pattern of 2-5 Hz.[38]

Visual pattern analysis combined with CSA proved better than univariate analysis with either of these methods alone, resulting in localization of the ictal onset in the mesial aspect of the temporal lobe in $>50 \%$ of patients. This was not significantly different from the presence of mesial sclerosis shown in MRIs.

Interictal brain SPECT scans showed lateralization of interictal brain hypoperfusion coinciding with the ictal onset zone in $92.3 \%$ of patients studied, and, in $5 \%$ of cases, hypoperfusion extending to the frontal lobe was found. There is evidence that the dysfunction may be several times greater than the observed structural defect.[39,40] This suggests that, in addition to anatomical abnormalities, a process of neuronal inhibition occurs during the interictal phase that translates into perfusion and metabolic dysfunction. We reported a higher coincidence than that reported by other authors, due, we believe, to a semi-quantitative analysis of brain perfusion in the regions evaluated. For example, in a 1991 study of 51 patients with refractory complex partial seizures, Rowe et al. found hypoperfusion in SPECT scans in 39\% of the 46 patients with a unilateral focus, for a positive predictive value of $86 \%$.[41]

Chance observations associated with functional MRI and SPECT have provided anecdotal evidence of an increase in regional brain blood flow in the epileptic temporal lobe minutes before ictal EEG onset, which suggests that changes observed in functional MRI and perictal SPECT cannot be explained solely as the results of ictal electroencephalographic activity, but rather as reflections of a change in neuronal activity that precipitates the transition from the interictal to the ictal state.[42-44] In any case, a detectable reduction of NAA in the affected hippocampus is not always accompanied by the corresponding atrophy, which is likely the consequence of a neuronal loss compensated in terms of cell density by reactive astrogliosis.[45,46]

We would emphasize that interictal and ictal studies complement each other. Lateralizing information provided by interictal SPECT in TLE patients, using semi-quantitative analytical methods, yielded results as significant as those yielded by ictal SPECT.

\section{CONCLUSIONS}

This study confirmed that localizing data provided by V-EEG and complemented by neurofunctional imaging studies can be used to perform successful temporal lobectomies on patients with drugresistant TLE and MRIs appearing normal or showing bilateral morphological abnormalities. As a result, CIREN introduced a strategy for the surgical treatment of patients with drug-resistant focal epilepsy in Cuba, thereby improving the cost-effectiveness of epilepsy surgery and significantly improving those patients' quality of life. -1 -

\section{REFERENCES}

1. Wiebe S, Blume WT, Girvin JP, Eliasziw M. A randomized, controlled trial of surgery for temporallobe epilepsy. N Engl J Med. 2001;345:311-8.

2. Deacon C, Wiebe S, Blume WT, McLachlan RS, Young GB, Matijevic S. Seizure identification by clinical description in temporal lobe epilepsy: How accurate are we? Neurology. 2003;61:1686-9.

3. Wieser HG. Mesial temporal lobe epilepsy versus amygdalar epilepsy: late seizure recurrence after initially successful amygdalotomy and regained seizure control following hippocampectomy. Epileptic Disord. 2000;2:141-52.

4. Li LM, Cendes F, Antel SB, Andermann F, Serles $W$, Dubeau F, et al. Prognostic value of proton magnetic resonance spectroscopic imaging for surgical outcome in patients with intractable temporal lobe epilepsy and bilateral hippocampal atrophy. Ann Neurol. 2000;47:195-200.

5. Hennessy MJ, Elwes RD, Honavar M, RabeHesketh S, Binnie CD, Polkey CE. Predictors of outcome and pathological considerations in the surgical treatment of intractable epilepsy associated with temporal lobe lesions. J Neurol Neurosurg Psychiatry. 2001;7:450-8.

6. Wieser HG, Blume WT, Fish D, Goldensohn E, Hufnagel A, King D, et al. ILAE Commission Report. Proposal for a new classification of outcome with respect to epileptic seizures following epilepsy surgery. Epilepsia. 2001;42:282-6.

7. Spencer S, Huh L. Outcomes of epilepsy surgery in adults and children. Lancet Neurol. 2008 Jun;7(6):525.
8. Schwartz TH, Marks D, Pak J, Hill J, Mandelbaum DE, Holodny Al, et al. Standardization of amygdalohippocampectomy with intraoperative magnetic resonance imaging: preliminary experience. Epilepsia. 2002;43:430-6.

9. Sun ZR, Kim MA, Heo KCh, Min K. Relationship between bilateral temporal hypometabolism and EEG findings for mesial temporal lobe epilepsy: Analysis of (18)F-FDG PET using SPM. Seizure. 2006;15(1):56-63.

10. Kim JH, Im KC, Kim JS, Lee SA, Lee JK, Khang SK, et al. Ictal hyperperfusion patterns in relation to ictal scalp EEG patterns in patients with unilateral hippocampal sclerosis: a SPECT study. Epilepsia. 2007 Feb;48(2):270-7. 
11. Yilmazer-Hanke DM, Wolf HK, Schramm J, Elger CE, Wiestler OD, Blumcke I. Subregional pathology of the amygdala complex and entorhinal region in surgical specimens from patients with pharmacoresistant temporal lobe epilepsy. J Neuropathol Exp Neurol. 2000;59:907-20.

12. Pitkanen A, Sutula TP. Is epilepsy a progressive disorder? Prospects for new therapeutic approaches in temporal-lobe epilepsy. Lancet Neurol. 2002;1:173-81.

13. Bernasconi A, Martinez V, Rosa-Neto $P$, D'Agostino D, Bernasconi N, Berkovic S, et al. Surgical resection for intractable epilepsy in "double cortex" syndrome yields inadequate results. Epilepsia. 2001;42:1124-9.

14. Engel Jr J. Mesial temporal lobe epilepsy: what have we learned? Neuroscientist. 2001;7:340-52.

15. Devinsky O. Diagnosis and treatment of temporal lobe epilepsy. Rev Neurol Dis. 2004;1(1):2-9

16. Engel Jr J. Finally, a randomized, controlled trial of epilepsy surgery. $\mathrm{N}$ Engl $\mathrm{J}$ Med. 2001;345:365-7.

17. Prevedello DM, Sandmann MC, Ebner A. Prognostic factors in mesial temporal lobe epilepsy surgery. Arq Neuropsiquiatr. 2000;58:207-13.

18. Alarcón G, Kissani N, Dad M, Elwes RD, Ekanayake J, Hennessy $\mathrm{MJ}$, et al. Lateralizing and localizing values of ictal onset recorded on the scalp: evidence from simultaneous recordings with intracranial foramen ovale electrodes. Epilepsia. 2001;42:1426-37

19. Pacia S, Devinsky O, Perrine K, Ravdin L, Luciano $D$, Vázquez $B$, et al. Clinical features of neocortical temporal lobe epilepsy. Ann Neurol. 1996;4:724-30.

20. Foldvary N, Nashold B, Mascha E, Thompson EA, Lee N, McNamara JO, et al. Seizure outcome after temporal lobectomy for temporal lobe epilepsy: a Kaplan-Meier survival analysis. Neurology. 2000;54:630-4.

21. Zijlmans M, Huiskamp GM, van Huffelen AC, Spetgens WP, Leijten FS. Detection of temporal lobe spikes: Comparing nasopharyngeal, cheek and anterior temporal electrodes to simultaneous subdural recordings. Clin Neurophysiol. 2008 Aug;119(8):1771-7.

22. Pacia SV, Ebersole JS. Intracranial EEG in temporal lobe epilepsy. J Clin Neurophysiol. 1999;16:399-407

23. Blanke O, Lantz G, Seeck M, Spinelli L, Grave DP, Thut G, et al. Temporal and spatial determination of EEG-seizure onset in the frequency domain. Clin Neurophysiol. 2000;111:763-72.

24. Ragazzo PC, Galanopoulou AS. Alfentanilinduced activation: a promising tool in the presurgical evaluation of temporal lobe epilepsy patients. Brain Res Rev. 2000;32:316-27.

25. Bosch-Bayard J, Valdés-Sosa P, Virues-Alba T, Aubert-Vázquez E, John ER, Harmony T, et al. 3D statistical parametric mapping of EEG source spectra by means of variable resolution electromagnetic tomography (VARETA). Clin Electroencephalogr. 2001;32(2):47-61.
26. Weder BJ, Schindler K, Loher TJ, Wiest R, Wissmeyer M, Ritter $\mathrm{P}$, et al. Brain areas involved in medial temporal lobe seizures: a principal component analysis of ictal SPECT data. Hum Brain Mapp. 2006 Jun;27(6):520-34.

27. Lamy $C$, Derambure $P$, Josien $E$, Destee $A$, Vermesch $\mathrm{P}$, Leduc $\mathrm{V}$, et al. Factors playing a role in the increase of localized perfusion observed by single-photon emission-computed tomography during an epileptic seizur. Rev Neurol (Paris). 2000;156:1113-9.

28. Holmes MD, Miles AN, Dodrill CB, Ojemann GA, Wilensky AJ. Identifying potential surgical candidates in patients with evidence of bitemporal epilepsy. Epilepsia. 2003;44:1075-9

29. Spencer SS. When should temporal-lobe epilepsy be treated surgically? Lancet Neurol. 2002;1:375-82.

30. Hardy SG, Miller JW, Holmes MD, Born DE, Ojemann GA, Dodrill CB, et al. Factors predicting outcome of surgery for intractable epilepsy with pathologically verified mesial temporal sclerosis. Epilepsia. 2003;44:565-8.

31. So EL. Integration of EEG, MRI, and SPECT in localizing the seizure focus for epilepsy surgery. Epilepsia. 2000;41 Suppl 3:S48-54.

32. Salanova $\mathrm{V}$, Andermann $\mathrm{F}$, Rasmussen $\mathrm{T}$, OIivier A, Quesney LF. Parietal lobe epilepsy. Clinical manifestations and outcome in 82 patients treated surgically between 1929 and 1988. Brain. 1995;118:607-27.

33. Quesney LF. Preoperative electroencephalographic investigation in frontal lobe epilepsy: electroencephalographic and electrocorticographic recordings. Can J Neurol Sci. 1991;18:559-63.

34. Chang BS, Ives JR, Schomer DL, Drislane FW. Outpatient EEG monitoring in the presurgical evaluation of patients with refractory temporal lobe epilepsy. J Clin Neurophysiol. 2002;19:152-6.

35. Battiston JJ, Darcey TM, Siegel AM, Williamson PD, Barkan HI, Akay M, et al. Statistical mapping of scalp-recorded ictal EEG records using wavelet analysis. Epilepsia. 2003;44:664-72.

36. Quesney LF, Risinger MW, Shewmon DA. Extracranial EEG evaluation. In: Surgical treatment of the epilepsies. New York: Raven Press; 1993. p. 173-95.

37. Risinger MW, Engel J Jr, Van Ness PC, Henry TR, Crandall P. Ictal localization of temporal lobe seizures with scalp/sphenoidal recordings. Neurology. 1989;39:1288-93.

38. Ebersole JS, Pacia S. Localization of Temporal Lobe Foci by Ictal EEG Patterns. Epilepsia. 1996;37:386-99.

39. Nelissen N, Van Paesschen W, Baete K, Van Laere K, Palmini A, Van Billoen $\mathrm{H}$, et al. Correlations of interictal FDG-PET metabolism and ictal SPECT perfusion changes in human temporal lobe epilepsy with hippocampal sclerosis. Neuroimage. 2006 Aug 15;32(2):684-95.

40. Andraus ME, Cosenza CA, Fonseca LM, Andraus CF, D'Andrea I, Alves-Leon SV. Interictal brain
SPECT in patients with medically refractory temporal lobe epilepsy. Arq Neuropsiquiatr. 2002;60:779-87

41. Rowe CC, Berkovic SF, Austin MC, Saling $M$, Kalnins RM, McKay WJ, et al. Visual and quantitative analysis of interictal SPECT with technetium-99m-HMPAO in temporal lobe epilepsy. J Nucl Med. 1991;32:1688-94.

42. Chang DJ, Zubal IG, Gottschalk C, Necochea A, Stokking R, Studholme C, et al. Comparison of statistical parametric mapping and SPECT difference imaging in patients with temporal lobe epilepsy. Epilepsia. 2002;43:68-74.

43. Van Paesschen W, Dupont P, Van Driel G, Van Billoen $\mathrm{H}$, Maes A. SPECT perfusion changes during complex partial seizures in patients with hippocampal sclerosis. Brain. 2003;126:1103-11.

44. McNally KA, Paige AL, Varghese $G$, Zhang $H$, Novotny EJ Jr, Spencer SS, et al. Localizing value of ictal-interictal SPECT analyzed by SPM (ISAS). Epilepsia. 2005 Sep;46(9):1450-64.

45. Leite CC, Lucato LT, Sato JR, Valente KD, Otaduy MC. Multivoxel proton MR spectroscopy in malformations of cortical development. Am J Neuroradiol. 2007;28(6):1071-5.

46. Breiter SN, Arroyo S, Mathews VP, Lesser RP, Bryan RN, Barker PB. Proton MR spectroscopy in patients with seizure disorders. Am J Neuroradiol. 1994;15:373-84.

\section{THE AUTHORS}

Lilia María Morales Chacón (Corresponding Author: lilia.morales@infomed. sld.cu), clinical neurophysiologist. Senior researcher and head of clinical neurophysiological services, brain SPECT laboratory, International Neurological Restoration Center (CIREN), Havana, Cuba.

Carlos Sánchez Catasús, nuclear physicist. Senior researcher and head of the brain SPECT laboratory, International Neurological Restoration Center (CIREN), Havana, Cuba.

Juan E. Bender, neurologist. Senior researcher and head of the adult static lesions clinic, International Neurological Restoration Center (CIREN), Havana, Cuba.

Jorge Bosch Bayard, specialist in health information technologies. Senior researcher. Cuban Neuroscience Center (CNEURO), Havana, Cuba.

María E García, neuropsychologist. Associate researcher, children's neurology clinic, International Neurological Restoration Center (CIREN), Havana, Cuba.

Ivan García Maeso, neurosurgeon. Adjunct researcher, International Neurological Restoration Center (CIREN), Havana, Cuba. 
Lourdes Lorigados Pedre, Senior researcher and head of the chemical neuroimmunology laboratory, International Neurological Restoration Center (CIREN), Havana, Cuba.

Bárbara Estupiñán Diaz, neuropathologist. Associate researcher, Neuropathology Department, International Neurological Restoration Center (CIREN), Havana, Cuba.

Otto Trápaga Quincoses, clinical neurophysiologist, neurophysiological services, International Neurological Restoration Center (CIREN), Havana, Cuba.
Margarita Báez Martín, clinical neurophysiologist. Associate researcher, neurophysiological services, International Neurological Restoration Center (CIREN), Havana, Cuba.

Abel Sánchez Coroneaux, practical nurse. Neurophysiological services, International Neurological Restoration Center (CIREN), Havana, Cuba.

Digna Pérez Madrigal, intensive care nurse. Telemetry Unit, International Neurological Restoration Center (CIREN), Havana, Cuba.
Miriam Guevara, licensed practical nurse. Telemetry Unit, International Neurological Restoration Center (CIREN), Havana, Cuba.

Marilyn Zaldívar Bermúdez, biological process technician. Neurophysiological services, International Neurological Restoration Center (CIREN), Havana, Cuba.

Ángel Águila, nuclear medicine technician. Brain SPECT laboratory, International Neurological Restoration Center (CIREN), Havana, Cuba.

Submitted: January 5, 2008

Approved for publication: June 18, 2008



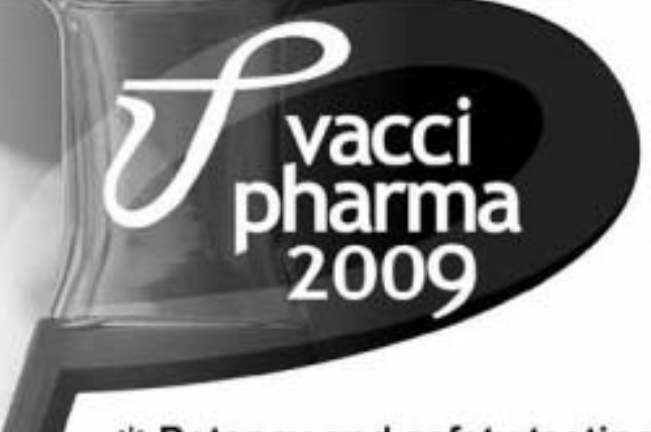

\section{* Potency and safety testing}

\section{* Clinical trials for preventive vaccines}

\section{* Therapeutic vaccine development}

Send abstracts to mlandys@finlay.edu.cu by February 15, 2009

For more information: www.scf.sld.cu/vaccipharma2009/vaccipharma09.htm 